\title{
FG Sagittae
}

\author{
Detlef Schönberner \\ Astrophysikalisches Institut Potsdam, An der Sternwarte 16, \\ D-14482 Potsdam, Germany \\ C. Simon Jeffery \\ Armagh Observatory, College Hill, Armagh BT61 9DG, \\ Northern Ireland
}

\begin{abstract}
FG Sagittae has evolved from a hot central star of a planetary nebula to an K-type supergiant within approximately the last 100 years. The generally accepted interpretation for this redward evolution is that of a late thermal pulse during the planetary nebula stage, wherein helium burning at the surface of the electron-degenerate carbon-oxygen core is reignited. As the star expands in response to the energy released by helium burning, envelope convection digs deeper and deeper until nuclearly processed material may get dredged-up to the stellar surface. Analysing the spectra as FG Sge is evolving would then give unique information about the temporal development of mixing processes occurring inside the star that are otherwise impossible to obtain. The existing abundance analyses do not give, however, a consistent picture. Especially the question about FG Sge's hydrogen abundance is still unsettled. We present a critical assessment of all the existing data, trying to find a self-consistent picture of the evolution of FG Sge, based on the latest evolutionary models.
\end{abstract}

\section{Introduction}

A unique object for the study of stellar evolution beyond the asymptotic giant branch (AGB) is FG Sge, a $9^{\text {th }}$ magnitude variable supergiant and the central star of the planetary nebula He 1-5. This object has several quite remarkable properties:

- It is known to have evolved from a rather hot object to a cool supergiant within half of a century, i.e. from spectral type B 4 in 1955 to K 2 in 1991 (cf. Herbig \& Boyarchuk 1968; van Genderen 1994). Since then the cooling has stopped at an effective temperature between 5000 and $6000 \mathrm{~K}$ (van Genderen \& Gautschy 1995). FG Sge had already brightened since 1900 when it was as faint as the $14^{\text {th }}$ magnitude.

Only two other objects, also central stars of planetary nebulae, exist with comparable properties: V4334 Sgr (Sakurai's object) covered a similar evolution in about only 5 years to become, in 1999, a carbon-rich giant, 
and V605 Aql which, after a brief excursion to the giant domain during the 1920's, is now a high-temperature [WC]-type central star.

- The temperature drop of FG Sge has been accompanied by a change of surface spectral signatures: Langer, Kraft, \& Anderson (1974) reported an increase of s-process element abundances between 1969 and 1972, and in 1981 the existence of $\mathrm{C}_{2}$ bands was noted (Acker, Jaschek, \& Gleizes 1981), indicating that FG Sge is carbon rich as well.

- Since 1992 occasional fadings of 3 to 4 magnitudes in $V$ have occurred, probably caused by the ejection of carbon-rich dust clouds in the line-ofsight and similar to those of $\mathrm{R} \mathrm{CrB}$ stars. This kind of erratic fading is also known for carbon-rich Miras (Whitelock 2000).

- Employing the reddening vs. distance diagram of Herbig et al. (1965), Herbig \& Boyarchuk (1968) found $d=2500 \mathrm{pc}$ and concluded that FG Sge is about 3 magnitudes fainter than a normal supergiant. It must thus be a low-mass object, as one would expect for a planetary nebula central star.

- Further information comes from the nebula itself: from its size and expansion velocity $\left(34 \mathrm{~km} \mathrm{~s}^{-1}\right)$ one estimates a kinematical post-ejection age of about 6000 years (Flannery \& Herbig 1973). The nebular ionization and excitation state indicates, considering the present electron density, that FG Sge must have achieved effective temperatures between 50000 and $75000 \mathrm{~K}$ within the last 500 years (Harrington \& Marionni 1976). Tylenda (1980) does not exclude a temperature as high as $100000 \mathrm{~K}$.

The generally accepted view is that FG Sge (and also V4334 Sgr and V605 Aql) are in the aftermath of a helium-shell flash that has occurred at the surface of the electron-degenerate carbon-oxygen core during the planetary nebula stage while the star is contracting to become a white dwarf. The uniqueness of this object lies in the fact that it provides us with information about the very last active phases of stellar evolution that are otherwise difficult or impossible to obtain.

For example, the very instance of the helium-shell flash indicates that FG Sge left the AGB while still powered by shell hydrogen burning (Schönberner 1981). Also, any evolution of the surface abundance would provide us with extremely valuable information about the nucleosynthesis occurring deep inside thermally pulsing AGB stars and of convective mixing processes during a thermal pulse.

In this context the most important questions are: i) Are the surface layers of FG Sge hydrogen-deficient, and if they are, when did they become hydrogendeficient? ii) What is the history of the surface s-process element enhancement? An answer to these fundamental questions is extremely difficult to obtain since any chemical composition changes are expected to occur during concomitant changes of the photospheric temperature. Moreover, the existing studies consider different epochs and use different datasets and different methods for the abundance analyses. In this review, we report an attempt to reassess the existing data supported by the latest evolutionary models in order to find a self-consistent picture of FG Sge. The literature is so extensive that it is impossible to consider all existing publications that deal with this particular object. Rather we 

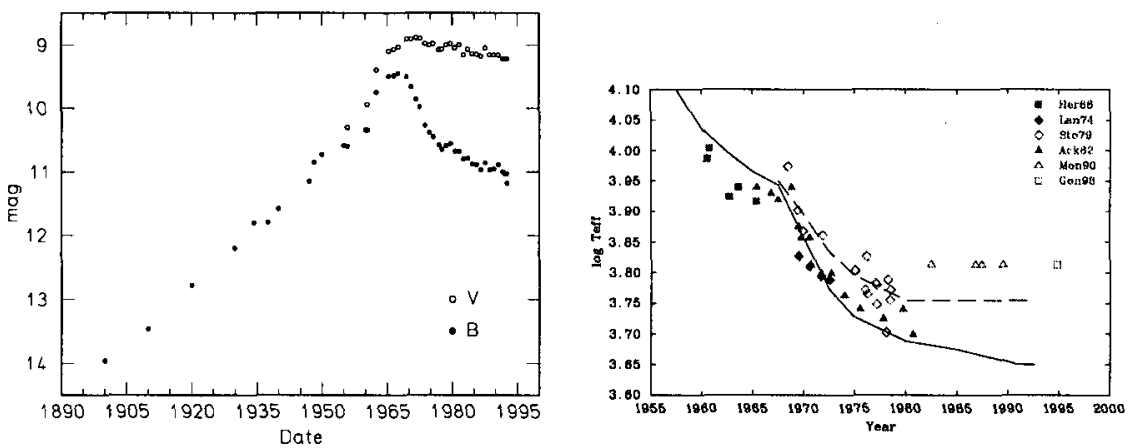

Figure 1. Left: Observed apparent magnitudes $V$ and $B\left(=m_{\mathrm{pg}}\right)$ of FG Sge from van Genderen \& Gautschy (1995, Table 3). Right: The run of $T_{\text {eff }}$ for FG Sge as determined spectroscopically (symbols) and (spectro)photometrically (van Genderen \& Gautschy 1995). Both low (solid) and high (dashed) $T_{\text {eff }}$ scales are given. The spectroscopic measurements are from Herbig \& Boyarchuk (1968, Her68), Langer et al. (1974, Lan74), Stone (1979, Sto79), Acker et al. (1982, Ack82), Montesinos et al. (1990, Mon90), and Gonzales et al. (1998, Gon98).

concentrate on those that are, in our opinion, most relevant for the questions posed above.

\section{The Expansion of FG Sge}

The most extensive account of FG Sge's evolution in brightness, spectral type, colour and pulsational period during the last 100 years has been given by van Genderen (1994) and van Genderen \& Gautschy (1995). Whereas the light curve indicates an average brightening of about $0.07 \mathrm{mag} \mathrm{yr}^{-1}$ up to $1965 \ldots 1970$ (Fig. 1, left), the determination of the effective temperature is much more difficult to establish. Broad-band photometry, spectrophotometry and spectroscopy have been used to measure $T_{\text {eff }}$ for FG Sge with rather limited success, as Fig. 1 (right) demonstrates. There are several reasons for this: intrinsic (pulsational) variability, blocking by the many lines from s-process elements, and possible dust emission in the infrared spectral region. Simultaneous UV, visual and IR photometry is necessary but does not exist. A more detailed discussion of the different temperature determinations will be given by Jeffery \& Schönberner (in preparation).

From the existing data, two $T_{\text {eff }}$ scales seem to emerge for FG Sge: a lowtemperature and a high-temperature scale. Since the latter is mainly based on spectral scans and IR photometry which are both prone to be disturbed by thermal dust emission, the low-temperature scale is to be preferred (cf. van Genderen \& Gautschy 1995). However, from both scales it is evident that the rapid cooling $\left(\approx 300 \mathrm{~K} \mathrm{yr}^{-1}\right.$ ) had virtually stopped by the 1980 's.

To reconstruct the past evolution of FG Sge in the Hertzsprung-Russell diagram, van Genderen \& Gautschy (1995) used the following assumptions, the 


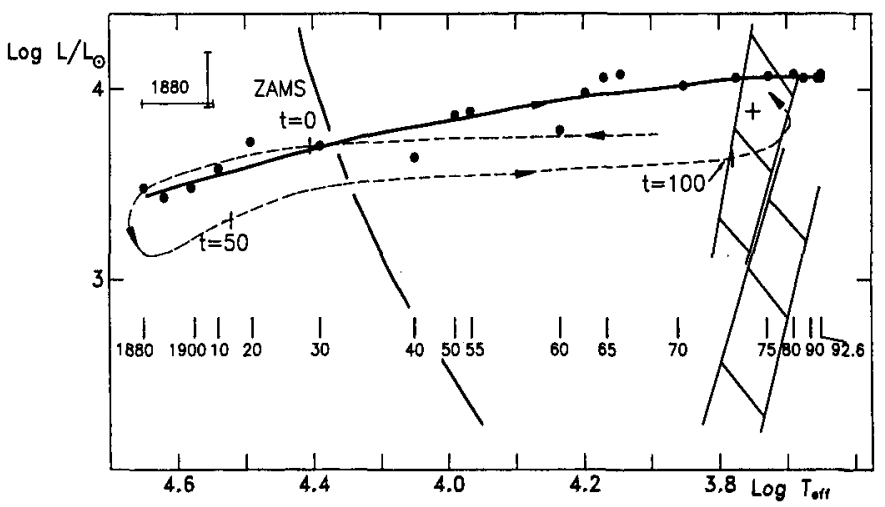

Figure 2. Hertzsprung-Russell-diagram presented by van Genderen \& Gautschy (1995): The solid line shows the reconstructed and smoothed redward track of FG Sge, with the corresponding dates indicated below. The dashed line indicates the evolutionary loop of a $0.6 \mathrm{M}_{\odot}$ post-AGB model that experienced a helium-shell flash at $t=0$ (Iben 1984). Also given are the ZAMS and the instability strips for Pop. I and II Cepheids.

low-temperature scale, a distance of $2500 \mathrm{pc}$ a mass of $0.8 \mathrm{M}_{\odot}$, and a light minimum at 1880 for a spectral type $\mathrm{O} 3(\approx 45000 \mathrm{~K})$. The stellar radius increased from $1 \mathrm{R}_{\odot}$ to $180 \mathrm{R}_{\odot}$ in 1990 , corresponding to a mean rate of $1.6 \mathrm{R}_{\odot} \mathrm{yr}^{-1}$, whereas the effective temperature dropped to $4500 \mathrm{~K}$. This track is shown in Fig. 2 and compared with a thermally-pulsing post-AGB model of $0.6 \mathrm{M}_{\odot}$ computed by Iben (1984). This model had left the AGB while still powered by hydrogen burning, and it happened that the next (= final) helium-shell flash occurred at $T_{\text {eff }} \simeq 25000 \mathrm{~K}$, well before the point on the white-dwarf track at which both shells would have become extinct.

This model comes very close to FG Sge, especially as far as the time scale of the redward excursion is concerned. A more detailed comparison with a grid of thermally-pulsing post-AGB models of different masses has been made by Blöcker \& Schönberner (1997), and these authors estimated for FG Sge a mass of $0.61 \pm 0.04 \mathrm{M}_{\odot}$. FG Sge appears to be the direct observational proof for the existence of thermal pulses in double-shell stellar configurations.

\section{The Chemical Evolution of FG Sge}

The expansion of FG Sge has naturally changed its spectrum, and it has been argued that this is not purely due to the changing surface temperature, but that there have been concomitant changes of the photospheric chemical composition as well. It must be noted, however, that each study considers a different epoch and uses a different dataset. Equally important is the fact that the existing studies approach the abundance analysis with different methods and different underlying assumptions. It is clear that it is difficult to put any temporal changes of FG Sge's chemical composition on a firm basis. In this section we concentrate 
on the most relevant abundance studies only and draw attention to existing inconsistencies.

Herbig \& Boyarchuk 1968: The first quantitative study of the chemical evolution of FG Sge covers the period $1960-1965$. The results are based on a differential curve-of-growth analysis relative to $\alpha \mathrm{Cyg}$, and the following points are interesting:

i) The absence of He I lines from 1962 onwards places limits on the helium abundance in the sense that it cannot have been significantly larger than normal during this epoch.

ii) A mild excess of carbon ( $0.7 \mathrm{dex})$ and oxygen $(0.5 \mathrm{dex})$, and

iii) an apparent excess of cobalt and strontium (0.8 dex).

Assuming that the differential abundance analyses are secure, a modern analysis of $\alpha$ Cyg can be used (Albayrak 2000) to compute absolute abundances (after correcting for differences between $\alpha$ Cyg and the Sun), with the following results:

i) carbon is overabundant by 1 dex, with $\mathrm{C} / \mathrm{O}>1$,

ii) helium, nitrogen - iron and nickel are near normal, and

iii) strontium, zirconium, barium and europium are overabundant by from 1.1 dex (barium) to 2.1 dex (strontium).

It must be emphasized that large overabundances in s-process elements are already evident from 1960 onwards, well before the surface layers became convectively unstable and allowed mixing between surface and deeper layers.

Langer, Kraft, \& Anderson 1974: Sometime after 1967 the spectrum of FG Sge started to show abnormally strong lines of Y II, Zr II, Ce II, La II and other s-process species, and apparent abundances rose 1.4 dex above the solar value (Langer et al. 1974). Based on spectra covering the time span from 1965 to 1972 , the most interesting results are:

i) a drop to near-normal carbon abundance (low weight only);

ii) roughly constant $\mathrm{Ti}, \mathrm{Cr}$ and $\mathrm{Fe}$ abundance;

iii) significant increases in $\mathrm{Y}$ and $\mathrm{Zr}$;

iv) $\mathrm{La}, \mathrm{Ce}, \mathrm{Pr}, \mathrm{Nd}$ and $\mathrm{Sm}$ with $0.9-1.5$ dex overabundances.

The strong increase of s-process elements within a time span of a few years (!) has previously been interpreted as evidence for mixing of surface matter with s-process contaminated material deep inside the star. This idea is supported by the fact that at this particular time FG Sge's surface temperature drops below the value necessary for surface convection $\left(\log T_{\text {eff }}=3.9\right.$, van Genderen \& Gautschy 1995, Fig. 8).

There are, however, doubts whether this picture is correct:

i) The production site of the s-process elements is the hydrogen-free, carbon-rich intershell region, and any mixing towards the surface would also increase carbon and decrease hydrogen at the surface. The replacement of a substantial amount of hydrogen by helium and carbon within a few years would drastically change the appearance of all lines during the same time. This is not observed. Moreover, such a mixing process will not proceed on the convective turnover timescale, but instead on a much longer one, controlled only by the downward movement of the convection boundary (see discussion in Sect. 4.). At this particular epoch's 
effective temperatures, however, the envelope convection is not yet even deep enough as to reach the hydrogen-free layers (Iben 1984; Blöcker \& Schönberner 1997).

ii) The overabundances gained after the (alleged) mixing event, up to 1.5 dex, correspond nicely to the adjusted values found for the earlier epoch when FG Sge was still hotter.

Since Langer et al. do not provide equivalent widths, it is impossible to reproduce their results, but from what has been said here it appears very likely that FG Sge was already enriched with s-process elements before it left the AGB.

Kipper \& Kipper 1993: These authors reported the detection of $\mathrm{C}_{2}$ and conducted the first fine analysis based on a Kurucz model atmosphere with $T_{\text {eff }}=5500 \mathrm{~K}, \log g=1.0$. They confirmed the carbon overabundance $(0.8$ dex) and found also marked overabundances for the s-process elements (up to $3.3 \mathrm{dex}$ for $\mathrm{Yb}$ ). It is to be noted that they found:

i) a mild deficiency of the iron group elements,

ii) s-process abundances again in encouraging agreement with the (adjusted) Herbig \& Boyarchuk (1968) results: Zr (0.7 vs. $1.2 \mathrm{dex}), \mathrm{Ba}(1.3$ vs. $1.1 \mathrm{dex})$, and Eu (1.6 vs. $1.7 \mathrm{dex})$.

High-resolution spectra taken in 2000 and analysed with the same model atmosphere give essentially the same results (Kipper \& Klochkova 2001). The oxygen abundance, based on one single [O I] line, resulted in $\mathrm{C} / \mathrm{O}=$ 2.5. No serious hydrogen deficiency seemed apparent.

Gonzales et al. 1998: This paper contains the most elaborate and detailed model atmosphere analysis (Kurucz) to date. It is based on modern highresolution and high $\mathrm{S} / \mathrm{N}$ spectra. Using the Fe I and Fe II lines, $T_{\text {eff }}=$ $6500 \pm 400 \mathrm{~K}, \log g=2.0 \pm 0.5$ and a 3 dex enhancement of s-process elements were deduced. The weakness of the $H \alpha$ line, when compared to that of $\alpha$ Per, seems to indicate a significant hydrogen deficiency. There are, however, difficulties posed by this analysis:

i) 1 dex of the s-process element enhancement must have occurred between 1992 (Kipper \& Kipper 1993) and 1994;

ii) the analysis requires the star to have both heated and shrunk in radius since the observations reported by Acker et al. (1982) without changing in $V$ magnitude. The absurdity of such a contradiction makes it necessary to consider these abundance determinations most carefully, since they are extremely sensitive to both $T_{\text {eff }}$ and $\log g$. Moreover, the luminosity-tomass ratio deduced from the surface parameters, $\simeq 450$ (solar units), is far below the typical value for a post-AGB star, viz. $\simeq 10000$, making simple comparisons between line strengths extremely risky: the Balmer lines depend, for given temperature, on both the helium-to-hydrogen ratio and/or gravity. The parameters used by Kipper \& Kipper (1993) give a luminosity-to-mass ratio, 3300 , which is at the lower limit of what one would expect for a typical post-AGB object.

Hawley \& Miller 1978: Analyzing the nebular emission-line spectrum provides the only method to get information about the stellar surface abundances prior to the shell flash and possible mixing episodes. The Hawley 
\& Miller analysis proved that the pre-flash envelope composition was quite typical for a planetary nebula:

i) $\mathrm{He} / \mathrm{H}=0.12$;

ii) oxygen, nitrogen and sulphur close to solar;

iii) only neon enhanced by 0.7 dex.

Comparing the oxygen abundance with the carbon abundance found by Herbig \& Boyarchuk (1968), it is obvious that FG Sge was already carbonrich, with $\mathrm{C} / \mathrm{O} \simeq 3$, before it left the $\mathrm{AGB}$.

Taking all the existing analyses into account, it appears to us that there is no observational evidence of any brief mixing episode that might have changed the surface composition of FG Sge into a hydrogen-free and/or s-process element enriched one. In this context it should be noted that all existing abundance studies contain the implicit assumption of a normal helium-to-hydrogen ratio.

\section{Theory of AGB and post-AGB Evolution}

In the context of FG Sge's evolution we have to focus only upon the evolution of low- and intermediate-mass stars, i.e. upon those stars which evolve after central helium burning through the AGB to become, if their initial mass was not too small, central stars of planetary nebulae and, finally, white dwarfs. The evolution along the AGB is characterized by three important features:

1. thermal instabilities of the helium-burning shell (thermal pulses), followed by the $3 r d$ dredge-up;

2. penetration of the convective envelope into the hydrogen burning shell for more massive objects (hot bottom burning);

3. strong mass loss, driven by radiation pressure on dust, which terminates the AGB phase.

An example of the evolution of such stars is presented in Fig. 3 which shows the complete evolutionary calculation of an initially $3 \mathrm{M}_{\odot}$ star from the main sequence through all following stages (Blöcker 1995a,b). Mass loss occurs mainly during the thermal-pulse phase, abruptly terminates the AGB evolution and determines the final mass, $0.605 \mathrm{M}_{\odot}$, of the central star.

On the AGB the stellar structure can be described as follows. The very compact, hydrogen-exhausted core of mass $M_{\mathrm{H}}$ is surrounded by a very dilute, fully convective, hydrogen-rich envelope of mass $M_{\mathrm{e}}$. The hydrogen-burning shell is the interface between core and envelope, and its radius is, in the extreme case, only about $10^{-4}$ times that of the stellar surface! The core, including the shell sources, evolves independently from the envelope as long as the latter contains sufficient mass to maintain the temperatures necessary for nuclear reactions at its base by gravothermal energy release. The evolutionary path of an AGB star in the HR diagram, as shown in Fig. 3, is a consequence of the envelope's response to the growth in mass of the core: expansion of the envelope along the Hayashi border line in order to accommodate the increasing luminosity dictated by the core, and contraction at nearly constant luminosity when the envelope mass drops below a few percent of a solar mass, mainly by mass loss from the 


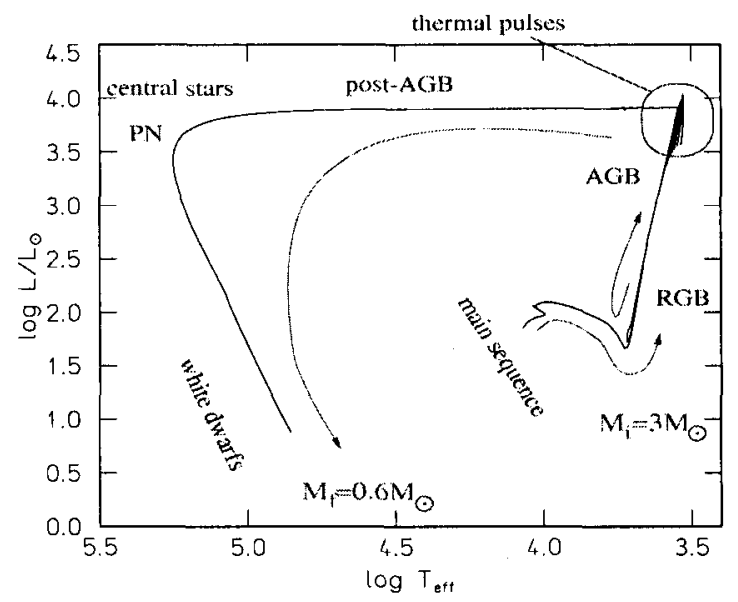

Figure 3. Complete evolutionary path of an initially $3 \mathrm{M}_{\odot}$ star from the main sequence to a white dwarf of $0.605 \mathrm{M}_{\odot}$ from Blöcker $(1995 \mathrm{a}, \mathrm{b})$, adapted by Herwig (priv. comm.). The main evolutionary phases are indicated (RGB = red giant branch, $\mathrm{AGB}=$ asymptotic giant branch, $\mathrm{PN}=$ planetary nebula), and the last thermal pulses (from a total of 17) at the tip of the AGB are clearly visible. The model is burning hydrogen until the shell source is extinguished shortly after the turning point at $T_{\text {eff }} \approx 160000 \mathrm{~K}$ is reached.

surface. When hydrogen burning cannot be sustained any longer, i.e. when the envelope mass falls below $\approx 10^{-4} \mathrm{M}_{\odot}$, the envelope shrinks rapidly to the (white dwarf) dimensions of the core, with a concomitant decrease of the luminosity (cf. Fig. 3). For the particular remnant mass of $0.6 \mathrm{M} \odot$, the whole evolution from the tip of the AGB to the white dwarf domain takes place in about 10000 years.

The most remarkable feature of the AGB evolution is a repeated instability of the helium-burning shell. During these so-called thermal pulses or helium-shell flashes the luminosity due to helium burning may exceed the surface luminosity by orders of magnitudes for a time span of about 100 years (Fig. 4, top). The full cycle length between two successive pulses depends sensitively on the core mass and is about 80000 years for $0.6 \mathrm{M}_{\odot}$. The large amount of energy liberated during the helium-shell instability drives a convective zone which mixes products of helium burning, i.e. carbon and oxygen, into the intershell region consisting primarily of CNO-cycled matter (Fig. 4, bottom left). Several hundred years after the helium luminosity peak the surface luminosity reaches its maximum. While hydrogen burning is extinct, the bottom of the envelope convection may penetrate into the carbon- and oxygen-enriched intershell layers and mix processed material into the envelope, the so-called $3 r d$ dredge-up. As the thermal disturbance declines, the envelope convection recedes, hydrogen re-ignites and burns again quiescently until the next pulse and its mixing episode. The repeated occurrence of shell flashes and the corresponding dredge-up of carbon during a star's AGB evolution may eventually turn the originally oxygen-rich 


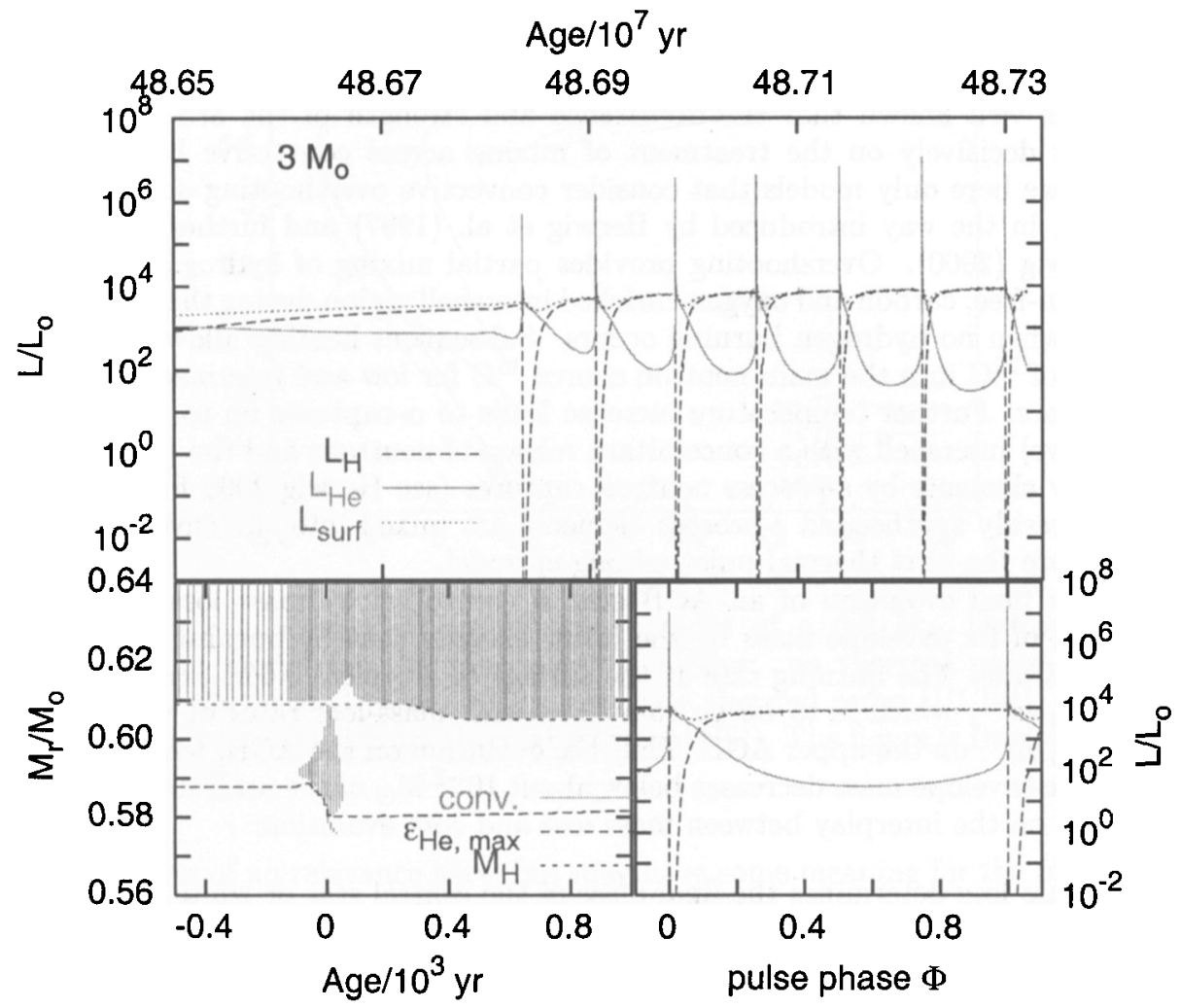

Figure 4. Luminosity and internal structure evolution of a $3 \mathrm{M}_{\odot}$ sequence according to Blöcker (2001). Top: Evolution of surface and hydrogen- and helium-shell luminosities during the first 7 thermal pulses. Bottom left: Extensions (mass wise) of convective regions around the burning region during the 6th pulse. $M_{\mathrm{H}}$ refers to the mass coordinate of the hydrogen-exhausted core, and $\epsilon_{\mathrm{He}}$ indicates the position with maximum energy production via helium burning. Time is set to zero at maximum helium luminosity, $L_{\mathrm{He}}$. The excess energy liberated during the helium-shell pulse creates a short-lived convective shell between $M_{\mathrm{r}}=0.58$ and $0.61 \mathrm{M}_{\odot}$, followed after about $400 \mathrm{yrs}$ by an extension of the envelope convection downwards to $M_{\mathrm{r}}=0.605 \mathrm{M}_{\odot}$, i.e. into completely hydrogen-free layers (3rd dredge-up). Bottom right: Luminosity evolution ( $\left.L_{\text {surf }}, L_{\mathrm{H}}, L_{\mathrm{He}}\right)$ vs. the thermal-pulse cycle phase, $\Phi$, for the complete 6 th pulse, lasting about 80000 years. Note that the left panel covers approximately only the phase range 0.995 to 0.01 . 
envelope into one with $\mathrm{C} / \mathrm{O}$ larger than unity, i.e. the spectral type will change from $\mathrm{M}$ to $\mathrm{C}$.

It is well known that the occurrence and strength of the 3rd dredge-up depends decisively on the treatment of mixing across convective boundaries. We utilize here only models that consider convective overshooting across these borders, in the way introduced by Herwig et al. (1997) and further described by Herwig (2000). Overshooting provides partial mixing of hydrogen into the hydrogen-free, carbon and oxygen enriched intershell region during the post-flash phases when no hydrogen burning occurs. Subsequent heating allows the conversion of ${ }^{12} \mathrm{C}$ into the main neutron source ${ }^{13} \mathrm{C}$ for low and intermediate-mass AGB stars. Further temperature increase leads to $\alpha$-captures on to ${ }^{13} \mathrm{C}$ in the (radiative) intershell with a concomitant release of neutrons and the production of heavy elements by s-process neutron captures (see Herwig 2000 for details). These freshly synthesized s-process elements are mixed into the envelope, but not before the next thermal-pulse mixing episode!

The final evolution of an AGB star is controlled by mass loss since the decrease of its envelope mass by the latter exceeds that by burning by orders of magnitude: The burning rate at the surface of a typical $0.6 \mathrm{M}_{\odot}$ core is only $10^{-7} \mathrm{M}_{\odot} \mathrm{yr}^{-1}$, which is to be be compared with mass-loss rates of more than $10^{-5} \mathrm{M}_{\odot} \mathrm{yr}^{-1}$ on the upper AGB. Thus the evolution off the AGB, which occurs when the envelope mass decreases below about $10^{-2} \mathrm{M}_{\odot}$ of the total stellar mass, depends on the interplay between mass loss and core evolution:

- mass loss determines the final mass of the central star or white dwarf;

- the internal structure corresponding to the phase of the thermal-pulse cycle at the end of the AGB determines the following evolution, viz. the hydrogen-burning, $\Phi>0.10$ ), or helium-burning, $\Phi<0.10$ ), mode (cf. Fig. 4, bottom right).

The evolution off the AGB occurs so rapidly compared with the thermalpulse cycle length that the phase does not change very much before the whitedwarf stage is reached and the shells are extinguished (Blöcker 1995b). Of relevance to the evolutionary history of FG Sge are hydrogen-burning AGB remnants with a thermal-pulse cycle phase sufficiently close to one, i.e. those that experience the next (and last) thermal pulse while they are contracting towards the white-dwarf stage (Schönberner 1979, Iben 1984). Three different possibilities can be distinguished (see Fig. 5).

1. The helium-shell flash occurs while the star is evolving along the horizontal part of the post-AGB evolution, well within the planetary-nebula regime (late thermal pulse).

2. The helium-shell flash occurs at the entrance to the white-dwarf cooling sequence when the burning shells are already extinct (very late thermal pulse).

3. There exists also a possibility that the flash occurs at the very top of the AGB when the envelope mass is already extremely small and the star about to leave the AGB, the asymptotic giant branch final thermal pulse. This 


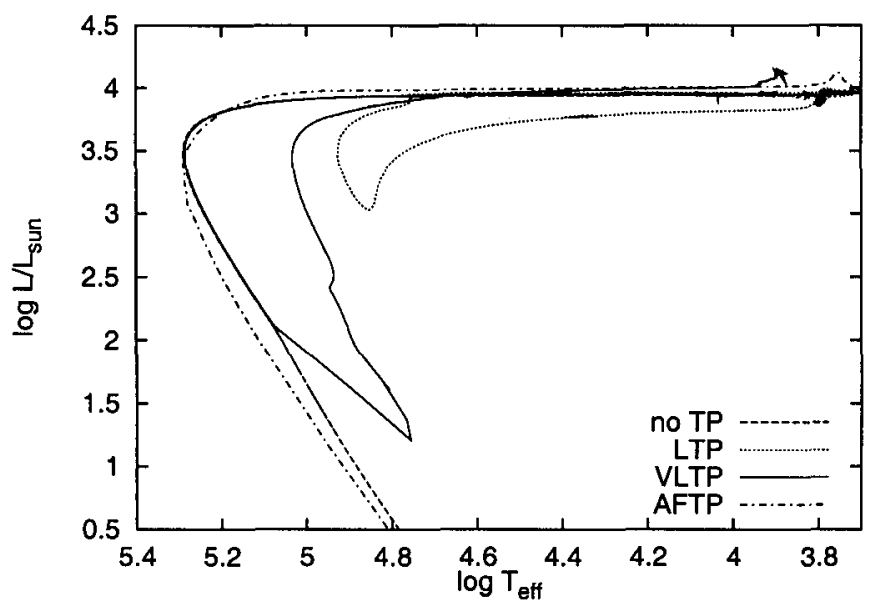

Figure 5. Post-AGB evolutionary tracks of a $0.6 \mathrm{M}_{\odot}$ hydrogenburning model for the four possible modes: no thermal pulse (no TP), late thermal pulse (LTP), very late thermal pulse (VLTP), and asymptotic-branch final thermal pulse (AFTP). The figure is from Herwig (2001).

case is of no relevance here, but may have some meaning for the formation of hydrogen-poor central stars (Herwig 2001).

In the first two cases, where the flash occurs far off the AGB, the star experiences a rapid expansion back to the vicinity of the AGB, sometimes called the $A G B$ born-again stage. The duration of this redward excursion is controlled by the gravitational-thermal timescale of the stellar envelope and is therefore rather short (cf. Blöcker \& Schönberner 1997).

The mixing properties of the LTP and VLTP models are relevant to the interpretation of the chemical evolution of FG Sge (Sect. 3.). The LTP case resembles the pulses on the AGB; shortly after the helium-shell luminosity peak, the bottom of the envelope convection moves across the helium-hydrogen discontinuity, and the 3rd dredge-up commences. This occurs when the reddest point of the loop is reached (corresponding to $\log T_{\text {eff }} \simeq 3.8$ in Fig. 5). But, contrary to the situation on the AGB, the amount of hydrogen-rich material in the stellar envelope is an order of magnitude smaller than that of the dredgedup hydrogen-free matter. About $5 \cdot 10^{-4} \mathrm{M}_{\odot}$ hydrogen-rich envelope matter is diluted by $4 \cdot 10^{-3} \mathrm{M}_{\odot}$ hydrogen-free intershell matter. The dredge-up is completed within about 200 years, corresponding to a mean dredge-up rate of hydrogen-free matter of $\simeq 2 \cdot 10^{-5} \mathrm{M}_{\odot} \mathrm{yr}^{-1}$. The final surface composition is $X \simeq 0.02, Y \simeq 0.4, X_{\mathrm{C}} \simeq 0.4, X_{\mathrm{O}} \simeq 0.18$ (Herwig 2001).

It should be noted that the mixing speed is entirely determined by the advance of the lower boundary of envelope convection, and hence ruled by stellar structure changes, not by convection properties. The increasing molecular weight and the decreasing opacity causes the envelope to shrink slowly during the dredge-up. It should also be noted that the kind of dredge-up described here occurs only if overshoot beyond convective boundaries is considered. 


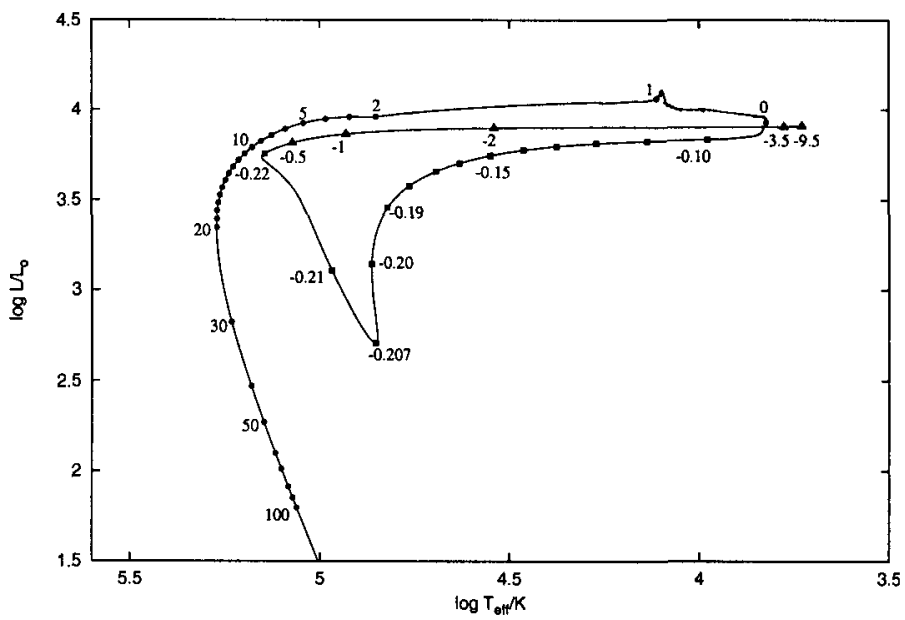

Figure 6. Evolutionary track of a $0.625 \mathrm{M} \odot$ post-AGB model suffering from a late thermal pulse (LTP). Symbols along the track give the time elapsed in units of $10^{3} \mathrm{yrs}$, with zero set to the minimum effective temperature after the helium shell flash. Age -3500 yrs corresponds to the beginning of the post-AGB evolution (Blöcker, Osterbart, \& Weigelt 2001).

The internal evolution is quite different in the VLTP case. This is the only known case where the flash-driven convective shell penetrates the outer hydrogen-rich layers and mixes hydrogen downwards towards the hot heliumburning layers. Hydrogen is partially burned and convectively mixed with hydrogen-free matter within a month. The stellar surface is virtually hydrogenfree and ${ }^{13} \mathrm{C}$-rich before the star evolves back to the AGB (Herwig et al. 1999; Herwig 2000).

Since FG Sge was definitively hydrogen-rich (and probably still is) when it was first observed, the VLTP case cannot be applied. It is rather a born-again object whose observational properties are consistent with the LTP scenario. The VLTP case may, however, be applicable to V4334 Sgr (Sakurai's object).

\section{An evolutionary scenario for FG Sge}

All the observational information available so far, weighted according to its credibility and combined with the latest evolutionary calculations, leads to the following likely evolutionary history of FG Sge (cf. also the Figs. 6 and 7):

Tip of AGB: FG Sge is a C-type AGB star with a significant production of s-process elements during previous thermal pulses. It departed from the AGB and formed a planetary nebula about 5000 years ago while still burning hydrogen (a so-called hydrogen burner). The final mass is close to $0.6 \mathrm{M}_{\odot}$ as is typical for central stars and white dwarfs. 


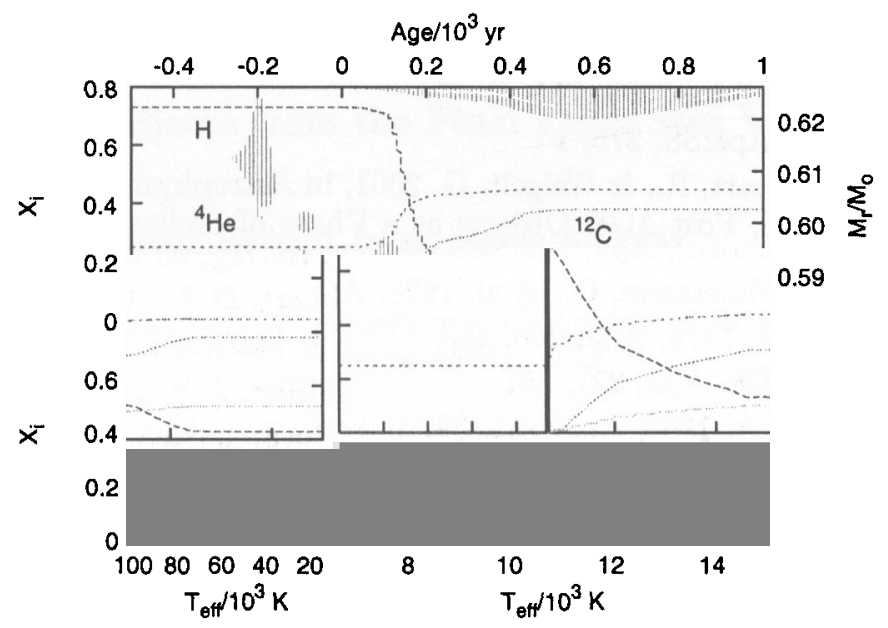

Figure 7. Top: Evolution of the surface abundances (left scale) and convective regions (shaded, right scale) for the model presented in Fig. 6. The hydrogen-rich envelope contains only about $2 \cdot 10^{-4} \mathrm{M}_{\odot}$. Bottom: Evolution of the surface abundances as a function of the effective temperature. The vertical line corresponds to age zero (top panel, and Fig. 6) and separates the redward and blueward branches of the evolutionary track shown in Fig. 6. - The figure is also from Blöcker et al. (2001).

Post-AGB: A late thermal pulse occurs about 100 to 200 years ago while FG Sge is still crossing the Hertzsprung-Russell diagram. A rapid return to the vicinity of the AGB follows. There is no dilution of the hydrogenrich envelope by hydrogen-free intershell matter.

Born-again AGB: There is a slow dilution of the envelope material by dredgeup with a rate of $\simeq 10^{-5} \mathrm{M}_{\odot} / \mathrm{yr}$, starting at the reddest point of the track (age $=0$ in Figs. 6 and 7). An extreme hydrogen deficiency is reached within less than 400 years, with a concomitant contraction towards $10000 \mathrm{~K}$ at a mean rate of $10 \mathrm{~K} \mathrm{yr}^{-1}$. FG Sge will continue its evolution as a [WC]-type central star since its surface composition is now $X=$ $0.05, Y=0.45, X_{\mathrm{C}}=0.38, X_{\mathrm{O}}=0.12$ (Fig. 7).

It should be noted that the time scales quoted above refer to only one evolutionary calculation. Details depend on the exact mass, on how the physics have been implemented and, especially, how overshooting is treated. Nevertheless we believe that we have presented a model for FG Sge that is consistent with its known properties.

\section{References}

Acker, A., Jaschek, M., \& Gleizes, F. 1982, A\&AS, 48, 363

Albayrak, B, 2000, A\&A, 364, 237 
Blöcker, T. 1995a, A\&A, 297, 727

Blöcker, T. 1995b, A\&A, 299, 755

Blöcker, T. 2001, Ap\&SS, 275, 1

Blöcker, T., Osterbart, R., \& Weigelt, G. 2001, in Astrophysics \& Space Science Library 265, Post-AGB Objects as a Phase of Stellar Evolution, ed. R. Szczerba \& S. K. Gorny (Dordrecht: Kluwer Acad. Publ.), 241

Blöcker, T., \& Schönberner, D. 1997, A\&A, 324, 991

Flannery, B. F., \& Herbig, G. H. 1973, ApJ, 183, 491

Gonzales, G., Lambert, D. L., Wallerstein, G., et al. 1998, ApJS, 114, 133

Harrington, J. P., \& Marionni, P. 1976, ApJ, 206, 458

Hawley, S. A., \& Miller, J. S. 1978, ApJ, 221, 851

Herbig, G. H., \& Boyarchuk, A. A. 1968, ApJ, 153, 397

Herbig, G. H., Preston, G. W., Smak, J., \& Paczynski, B. 1965, ApJ, 141, 617

Herwig, F. 2000, A\&A, 360, 952

Herwig, F. 2001, Ap\&SS, 275, 15

Herwig, F., Blöcker, T., Langer, N., \& Driebe, T. 1999, A\&A, 349, L5

Herwig, F., Blöcker, T., Schönberner, D., El Eid, M. F. 1997, A\&A, 324, L81

Iben, I. Jr. 1984, ApJ, 277, 333

Kipper, T, \& Kipper, M. 1993, A\&A, 276, 389

Kipper, T, \& Klochkova, V. G. 2001, Baltic Astronomy, 10, 393

Langer, G. E., Kraft, R. P., \& Anderson, K. S. 1974, ApJ, 189, 509

Montesinos, B., Cassatella, A., Gonzales-Riestra, R., et al. 1990, ApJ, 363, 245

Schönberner, D. 1979, A\&A, 79, 108

Schönberner, D. 1981, A\&A, 103, 119

Stone, R. P. S. 1979, PASP, 91, 389

Tylenda, R. 1980, Acta Astron., 30, 433

van Genderen, A. M. 1994, A\&A, 284, 465

van Genderen, A. M., \& Gautschy, A. 1995, A\&A, 294, 453

Whitelock, P. 2000, in IAU Symp. 177, The Carbon Star Phenomenon, ed. R. F. Wing (Dordrecht: Kluwer), 179 